\title{
Rosellinia sp.: saprophyte that becomes a parasite of cacao tree
}

\section{Abstract}

Rosellinia sp.: is a facultative parasite present in the main grow cacao regions from Colombia. Its importance is correlated with the climate being greater in the moderate warms. Until today there isn't a precise control method. Then, it is necessary to seek alternatives like to research the effect of some disinfectants applied on the soil like Formaldehyde, Sodium Hypoclorite among others. The Castor-oil is highly susceptible to the fungus and can to use like indicator plant of its persistence in the soil.
Volume 4 Issue 2 - 2020

\author{
Gabriel Cubillos \\ Agronomist Engineer, Colombia
}

Correspondence: Gabriel Cubillos, Agronomist Engineer, Colombia, Email cubillos.g@gmail.com

Received: March 05, 2020 | Published: April 22, 2020

\section{Introduction}

Rosellinia sp.: (Figure 1) is a natural soil fungus that for unknown reasons becomes an occasional cacao parasite. Its dissemination is silent causing the death of trees in different places within the crop (foci) but stopping its expansion sometime after leaving the affected areas. That means that it is not known why the fungus changes its condition from saprophyte to parasite and why it returns to its natural condition of saprophyte. It is reported in Central and South America, West Africa, West Indies and Asia. ${ }^{1}$

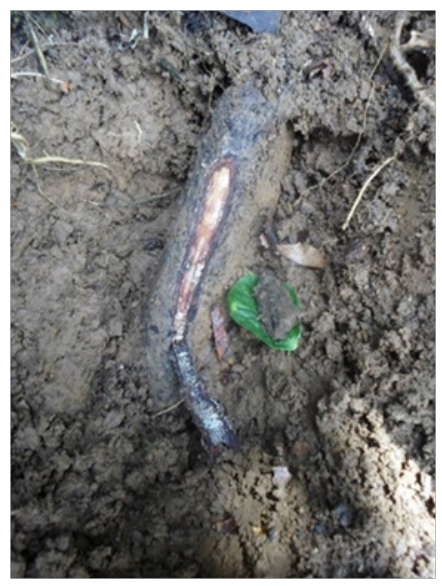

Figure I Rosellinia sp.: affecting a cacao root.

In Colombia it is registered in all producing cacao regions but its frequency and severity is higher in regions of moderate warm weather. The case of the La Margarita estate, municipality of Palestina, department of Caldas, located at 1,300 m.a.s.1, which registered an incidence of $2.2 \%$ spread over 13 foci, with a population of 25,000 seven-year-old hybrid cocoa trees that it is considered a relatively important incidence. ${ }^{2}$

An effective, cost-effective and easily accessible control method for the cacao producer in general has not yet been found.

\section{Hypothesis}

\section{Changing from saprophyte to parasite}

Rosellinia is a natural inhabitant of the soil. It can happen that the root of a tree suffers a trauma that causes its death, becoming a culture substrate to re-power the fungal structures that invade the entire root system until ending the life of the tree and spreading toward the trees more near.

\section{Changing from parasite to saprophyte}

The abandonment of the foci of Rosellinia implies changes in the conditions that favor the parasitism of the fungus and the progressive weeding of the affected area. This situation possibly determines the drastic reduction of decomposing organic matter that nourishes the fungus weakening its aggressiveness and consequently, its parasitism.

Simultaneously, weed species that cover the foci area have different resistant grades to the fungus preventing their spread. In this way, the fungus returns to its natural saprophyte condition.

\section{Control proposal}

It is essential to diagnose early the nature of the causative agent of the death of a tree and thus prevent the spread of the disease. If it is Rosellinia, it is possible to test the disinfection of the soil corresponding to the tree, after sweeping the litter that covers it, with a disinfectant such as Formaldehyde (initially testing the $10 \%$ dose) or $2.5 \%$ Sodium Hypochlorite. One week after disinfection, 8 seeds of Castor-oil (Ricinus communis), an indicator species of the parasitic presence of Rosellinia (Figure 2) are sown 1 meter away around the affected tree, and during a period of two months its monitoring is done to determine if the seedlings they grow healthy. If that is achieved, it means that the disinfection was successful. The following step is to re-plant the cacao trees died by the disease.

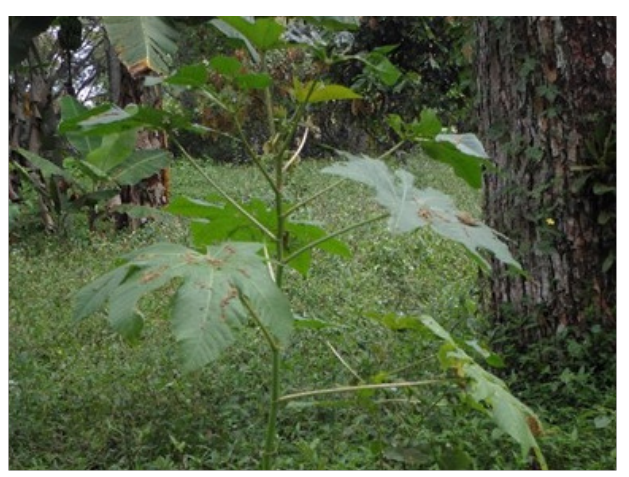

Figure 2 Castor-oil (Ricinus communis), a plant that indicates the parasitic presence of Rosellinia sp.: in a cacao area affected by the fungus. 
Proven the effectiveness of the disinfection, we would be face an easy and low-cost control method, accessible to small and large cocoa producers.

\section{Acknowledgments}

None.

\section{Conflicts of interest}

The authors declare have no conflict of interest about the publication of this paper.

\section{References}

1. Flood J, Hoopen t, Krauss U, et al. Root-infecting fungi attacking theobroma cacao. in: cacao diseases. a history of old enemies and new encounters. BA Bailey, LW Meinhardt, editors. Cham : Springer International Publishing. 2016;449-480.

2. Merchán VM. La Rosellinia del cacao. El cacaotero colombiano. 1990;13:13-19. 